\title{
Inequality Measurement for Ordered Response Health Data
}

\author{
Ramses H. Abul Naga* and Tarik Yalcin ${ }^{\dagger}$
}

\footnotetext{
$\$$ We are grateful to Pierre-Yves Geoffard for helpful comments. We also thank Gerard Antille for discussions.

${ }^{*}$ Institute for Health Economics and Management (Université de Lausanne).

${ }^{\dagger}$ Institute for Health Economics and Management (Université de Lausanne).
} 


\begin{abstract}
When health status is an ordered response variable, Allison and Foster (2004) postulate that a distribution $Q$. exhibits more inequality than a distribution $P$. if $Q$. is obtained from $P$. via a sequence of median preserving spreads. This paper introduces a parametric family of inequality indices which are founded on the Allison and Foster ordering.
\end{abstract}

Keywords: Self-reported health status, inequality orderings, inequality measures.

JEL codes: I3, I1. 


\section{Distributional Analysis Research Programme}

The Distributional Analysis Research Programme was established in 1993 with funding from the Economic and Social Research Council. It is located within the Suntory and Toyota International Centres for Economics and Related Disciplines (STICERD) at the London School of Economics and Political Science. The programme is directed by Frank Cowell. The Discussion Paper series is available free of charge. To subscribe to the DARP paper series, or for further information on the work of the Programme, please contact our Research Secretary, Leila Alberici on:

Telephone: UK+20 79556674

Fax: $\quad$ UK+20 79556951

Email: 1.alberici@lse.ac.uk

Web site: $\quad \underline{\text { http://sticerd.Ise.ac.uk/DARP }}$

(C) Authors: Ramses H. Abul Naga and Tarik Yalcin. All rights reserved. Short sections of text, not to exceed two paragraphs, may be quoted without explicit permission provided that full credit, including $(\mathcal{C}$ notice, is given to the source. 


\section{Introduction}

Policy reform in the health sector requires an evaluation of its likely impact on health inequality. Health data however differ from income data in several respects, making the body of tools available for the measurement of income inequality not fully suited for the analysis of health disparities. One such difference between health and income data is that income is a continuous variable, while data on self-reported health status [SRHS] are qualitative measurements. ${ }^{1}$

The context of SRHS data is somewhat different from that of income data, where transformations of summary statistics of location and dispersion (e.g. the mean, variance and other moments of the distribution) form the basis of an inequality index. Simple operations such as adding and averaging are however not available for qualitative data; thus the need to develop inequality indices tailored for SRHS data.

One solution to the problem raised by qualitative health data is to adopt a particular health scale, which exploits the ordered nature of subjective health responses. That is, we know that enjoying a good health is better than a poor health, and we may wish to assign numerical values (dictated by a particular health scale) to each available response. However, as Allison and Foster (2004) point out, conventional inequality indices (i.e. those developed for income data) will be sensitive to the particular scale used to convert subjective health responses to numerical values; i.e. small variations in the health scale may reverse the ordering of two distributions in relation to the Lorenz curve or a conventional inequality index.

Yet another solution to this problem consists in treating health status as a latent variable and SRHS data as ordered responses underlying this variable. An appropriate econometric methodology has thus been elaborated and used to estimate health status as a continuous variable (Wagstaff et al., 1991). A considerably different methodology for the analysis of SRHS data has recently been proposed by Allison and Foster: when health status is an ordered qualitative variable, these authors postulate that a distribution $Q$ exhibits more inequality than a distribution $P$ if $Q$ is obtained from $P$ via a sequence of median preserving spreads. The approach developed by these authors is thus ordinal: when the conditions

\footnotetext{
${ }^{1}$ There are also multiple dimensions to health status; the definition used to capture health status is thus not unique. There also exist more objective data on health status. However, as Strauss and Thomas (1998) point out, such data are costly to collect, and are less frequently encountered in practice.
} 
for a median preserving spread are satisfied, two distributions may be ordered. However, inequality indices providing numerical values to the underlying amount of inequality, the quantification of the underlying change in inequality etc., have yet to be fully explored in the present context.

In order to pin down the problem involved with converting qualitative responses into a numerical scale, consider the following example. Let individuals choose between three possible health states, say bad, fair and excellent. There are two frequency distributions: $\pi=\left[\begin{array}{lll}0.33 & 0.67 & 0.00\end{array}\right]$, and $\theta=\left[\begin{array}{lll}0.00 & 0.67 & 0.33\end{array}\right]$ which are the subject of our inequality comparisons. The two distributions differ in that under $\pi$ one third of the population judges itself as being in bad health, whereas under $\theta$ there is a comparable share assessing its health state as being excellent. In relation to the various states we shall define three alternative health scales: $c^{1}=\left[\begin{array}{lll}2 / 3 & 4 / 3 & 5 / 3\end{array}\right], c^{2}=\left[\begin{array}{lll}1 & 2 & 3\end{array}\right]$ and $c^{3}=\left[\begin{array}{lll}2 / 3 & 4 / 3 & 10 / 3\end{array}\right]$. Now if we define $\operatorname{var}(f ; c)$ as being the variance of frequency distribution $f$ under scale $c$, we obtain $\operatorname{var}\left(\pi ; c^{2}\right)=\operatorname{var}\left(\theta ; c^{2}\right)=0.222$. However, we also find 0.099 $=\operatorname{var}\left(\pi ; c^{1}\right)>\operatorname{var}\left(\theta ; c^{1}\right)=0.025$ while $0.099=\operatorname{var}\left(\pi ; c^{3}\right)<\operatorname{var}\left(\theta ; c^{3}\right)=0.899$. Thus the variance reverses the ranking of the two distributions according to which scale is adopted.

We mention two other problems in relation to the measurement of inequality for qualitative data, which our paper will address. Firstly, how do we enforce the requirement for an inequality index for qualitative data to take on a zero value when all individuals rank their health status identically? This desired property, known as the normalization axiom, is now required to hold for all increasing health scales. An additional problem is how to construct an inequality measure which allows for a convenient parametrization of different social judgments about inequality above and below the median, and at both ends of the distribution. As the above variance example serves to illustrate, the proposed inequality index cannot be made to be sensitive to the numerical values underlying each health state, since these numbers only serve to order the various outcomes, but are otherwise arbitrary.

This paper introduces a family of inequality indices which are founded on the Allison and Foster $[A F]$ ordering. Specifically,

- We examine the conditions under which the ordering of distributions by the inequality index is made invariant to the choice of health scale.

- We formalize the conditions under which the inequality index satisfies the normalization axiom in the context of qualitative data. 
- We characterize the entire of class of continuous inequality indices founded on the $A F$ ordering which satisfy the above normalization and invariance axioms.

- We propose a parametric family of inequality indices for SRHS data and derive specific indices within this class.

Regarding this last point, our parametric family of indices uses for unique data input the cumulative distribution function, and is thus operational without the adoption of any particular health scale. Apouey (2006) has independently proposed measures of polarization for SRHS data, also on the basis of the cumulative distribution function. While there exists some common ground between the axioms underlying inequality and polarization concepts, the related inequality and polarization indices are tailored to address separate questions, and are thus complementary in the analysis of SRHS data.

Some intuition for the approach developed in the paper may be obtained by examining Figure 1. Return to our hypothetical example whereby individuals choose between three possible health outcomes. One health scale $c^{a}$, assigns numerical values $c^{a} \doteq\left(c_{1}^{a}, c_{2}^{a}, c_{3}^{a}\right)$, whereas a second health scale $c^{b}$ assigns values $c^{b} \doteq\left(c_{1}^{b}, c_{2}^{b}, c_{3}^{b}\right)$ to the three health responses. The scales are entirely arbitrary, except that $c_{1}^{a} \leq c_{2}^{a} \leq c_{3}^{a}$ and likewise $c_{1}^{b} \leq c_{2}^{b} \leq c_{3}^{b}$. While changes in the scale stretch to varying degrees the width of the steps of the underlying cumulative distribution function $F\left(c_{i}\right)$, the height of the cumulative distribution function is invariant to scale changes provided the ordering of responses is maintained. Thus, the cumulative proportions of individuals underlying each health response will be the main input used to derive health inequality indices.

In Section 2 of the paper we introduce some definitions and terminology which will be used subsequently. In Section 3 we propose a set of axioms which an inequality index for SRHS data ought to verify and we characterize the family of indices which satisfy these axioms. We use this result to introduce in Section 4 a parametric family of inequality indices for SRHS data. In Section 5 we propose an alternative set of axioms and obtain a related family of inequality indices. Section 6 contains an illustrative example using data from a recent wave of the Swiss Health Survey. An appendix gathers proofs of our main results. 


\section{Definitions and examples}

We consider a situation whereby the health status of a person is measured according to a scale $c \doteq\left(c_{1}, \ldots, c_{n}\right)$. The scale is entirely arbitrary apart from the restriction $0<c_{1} \leq c_{2} \leq \ldots \leq c_{n}$. We denote $C \doteq\left\{c: 0<c_{1} \leq c_{2} \leq \ldots \leq c_{n}\right\}$ the set of ordered increasing scales. Because outcomes are ordinal, basic operations such as summations and calculations of summary statistics (mean and other moments) will not form the basis of our measurement of inequality. However, the proportions underlying each outcome, and the associated probability distribution function will be the inputs to our inequality indices.

Define $p_{i}$ as the proportion of individuals in the class $c_{i}$. We have that $0 \leq$ $p_{i} \leq 1$ and $\sum_{i=1}^{n} p_{i}=1$. We also define a frequency distribution $\pi \doteq\left(p_{1}, \ldots, p_{n}\right)$ and a cumulative distribution function $\Pi \doteq\left[\Pi\left(c_{1}\right), \Pi\left(c_{2}\right), \ldots, \Pi\left(c_{n}\right)\right]$. We thus have

$$
\Pi\left(c_{i}\right)=p_{1}+p_{2}+\ldots+p_{i} \doteq P_{i}
$$

such that $P_{1}=p_{1}, P_{n}=1$, and we write $\Pi=\left(P_{1}, \ldots, P_{n}\right)$. We also let $\Lambda$ denote the set of cumulative distribution functions, and let $\Theta \doteq\left(Q_{1}, \ldots, Q_{n}\right), \Pi$ and $\Upsilon$ be any three elements of $\Lambda$.

A partial ordering over the set $\Lambda$ is a relation $\prec$ which satisfies the following three properties:

$$
\begin{aligned}
& (P O 1) \Pi \prec \Pi(R E F L E X) \\
& (P O 2) \Pi \prec \Theta \text { and } \Theta \prec \Pi \text { implies } \Pi=\Theta(\text { ANTISYM }) \\
& (P O 3) \Pi \prec \Theta \text { and } \Theta \prec \Upsilon \text { implies } \Pi \prec \Upsilon(\text { TRANSI })
\end{aligned}
$$

The first property states that a distribution is as good as itself. The second property states that if $\Pi$ is preferred to $\Theta$ and the reverse is also true, then we must take it that the distributions $\Pi$ and $\Theta$ are identical. Finally, the third property implies some coherence in the preference relation over distributions. The Lorenz criterion for comparing income distributions is one such relation which satisfies the above three properties. Allison and Foster (2004) postulate that a distribution $\Theta$ exhibits more inequality than a distribution $\Pi$ if $\Theta$ is obtained from $\Pi$ via a sequence of median preserving spreads. Formally, the $A F$ criterion for ranking distributions is a relation $\prec_{A F}$ over the set $\Lambda$ which satisfies the following three properties:

$(A F 1) \Pi$ and $\Theta$ have identical median states $m$

$(A F 2)$ for all $j<m \quad P_{j} \leq Q_{j}$ 
(AF3) for all $j \geq m \quad P_{j} \geq Q_{j}$

Intuitively, $\Pi$ is more concentrated than $\Theta$ around the median state. Accordingly, $\Pi$ is more equally distributed than $\Theta$. The following result follows from the properties $[A F 1-A F 3]$ :

Lemma 1 The relation $\prec_{A F}$ is a partial ordering over the set of cumulative distribution functions $\Lambda$.

As such, there exists a function $\phi():. \Lambda \rightarrow R$ which preserves the partial ordering relation $\prec_{A F}$ of the lemma, and quantifies the level of dispersion underlying a distribution $\Pi$. Our task in this paper is to construct inequality indices around the function $\phi\left(P_{1}, \ldots, P_{n}\right)$.

Example 1 Let there be five health states and consider two frequency distributions $\pi^{*} \doteq(0.05,0.05,0.1,0.75,0.05)$ and $\theta^{*} \doteq(0.05,0.05,0.1,0.65,0.15)$. Then we have

$$
\begin{aligned}
& \Pi^{*}=\left[\begin{array}{lllll}
0.05 & 0.10 & 0.20 & 0.95 & 1
\end{array}\right] \\
& \Theta^{*}=\left[\begin{array}{lllll}
0.05 & 0.10 & 0.20 & 0.85 & 1
\end{array}\right]
\end{aligned}
$$

The median health state for both distributions is $m=4$. Furthermore, $\Pi^{*}$ and $\Theta^{*}$ satisfy the other two requirements $(A F 2)$ and $(A F 3)$, with $\Pi^{*} \prec_{A F} \Theta^{*}$. That is $\Pi^{*}$ is more equally distributed than $\Theta^{*}$.

\subsection{Two distributions}

We next partition a distribution $\Pi \in \Lambda$ into two line vectors $\Pi^{L}$ and $\Pi^{H}$ :

$$
\begin{aligned}
\Pi & \doteq\left[\begin{array}{ll}
\Pi^{L} & \Pi^{H}
\end{array}\right] \\
\Pi^{L} & \doteq\left[\begin{array}{llll}
P_{1} & P_{2} & \cdots & P_{m-1}
\end{array}\right] \\
\Pi^{H} & \doteq\left[\begin{array}{llll}
P_{m} & P_{m+1} & \cdots & P_{n}
\end{array}\right]
\end{aligned}
$$

Starting with a distribution $\Pi$ with median $m$, a sequence of equalizing median preserving transfers (the reverse operation from a median preserving spread) will ultimately produce a distribution

$$
\begin{aligned}
\hat{\Pi} & \doteq\left[\begin{array}{ll}
\hat{\Pi}^{L} & \hat{\Pi}^{H}
\end{array}\right] \\
\hat{\Pi}^{L} & \doteq\left[\begin{array}{lll}
0 & \cdots & 0
\end{array}\right] \\
\hat{\Pi}^{H} & \doteq\left[\begin{array}{lll}
1 & \cdots & 1
\end{array}\right]
\end{aligned}
$$


That is, within the context of the $A F$ ordering, $\hat{\Pi}$ is the most egalitarian distribution; i.e. it is not possible to define a distribution $\Pi^{+} \neq \hat{\Pi}$ such that $\hat{\Pi}$ is a median preserving spread of $\Pi^{+}$. Conversely, there exists a distribution

$$
\begin{aligned}
\check{\Pi} & \doteq\left[\begin{array}{ll}
\check{\Pi}^{L} & \check{\Pi}^{H}
\end{array}\right] \\
\check{\Pi}^{L} & \doteq\left[\begin{array}{lll}
\frac{1}{2} & \cdots & \frac{1}{2}
\end{array}\right] \\
\check{\Pi}^{H} & \doteq\left[\begin{array}{llll}
\frac{1}{2} & \cdots & \frac{1}{2} & 1
\end{array}\right]
\end{aligned}
$$

which is the least egalitarian distribution with median $m$. The frequency distribution associated with $\check{\Pi}$ is $\check{p} \doteq\left(\frac{1}{2}, 0, \ldots, 0, \frac{1}{2}\right)$ where half the population has the least favourable outcome and the other half of the population enjoys the highest possible state. Again, $\check{\Pi}$ is the least egalitarian distribution because it is not possible to define a distribution $\Pi^{-} \neq \check{\Pi}$ such that $\check{\Pi} \prec_{A F} \Pi^{-}$. The result stated below will prove useful in defining the normalization axiom in relation to inequality indices based on the $A F$ ordering.

Lemma 2 For all $\Pi \in \Lambda$ we have $\hat{\Pi} \prec_{A F} \Pi$ and $\Pi \prec_{A F} \check{\Pi}$.

$\hat{\Pi}$ is the analogue of the distribution where everyone has the mean endowment in the income inequality literature, associated with maximal equality; likewise $\check{\Pi}$ is the analogue of the distribution where one individual owns all the income and all other individuals have zero shares, associated with the minimal level of income equality.

An inequality index here will be defined as a function $I(\Pi, c): \Lambda \times C \rightarrow R_{+}$. Below we propose a family of indices which preserve the $A F$ ordering. By this it is meant that for any given health scale, if a distribution $\Theta$ is obtained from a distribution $\Pi$ via one or several median preserving spreads, then $\Theta$ is required to exhibit a higher level of inequality than $\Pi$ : $\Pi \prec_{A F} \Theta \rightarrow I(\Pi, c) \leq I(\Theta, c)$. The following example illustrates the order-preserving property.

Example 2 Returning to the distributions $\Pi^{*}$ and $\Theta^{*}$ of Example 1 we have established that $\Pi^{*} \prec_{A F} \Theta^{*}$. Now consider the inequality index ${ }^{2}$

$$
I_{A}(\Pi, c) \doteq 1-\left(\frac{2 \sum_{i=1}^{n}\left|P_{i}-0.5\right|-1}{n-1}\right)
$$

\footnotetext{
${ }^{2}$ The use of absolute deviations of the components of the cumulative distribution function from 0.5 is independently proposed by Apouey (2006) in order to measure the level of polarization in SRHS data.
} 
Then we have that $I_{A}\left(\Pi^{*}, c\right)=0.20$ while $I_{A}\left(\Theta^{*}, c\right)=0.25$. Furthermore for the limiting scenarios $\hat{\Pi}=(0,0,0,1,1)$ and $\check{\Pi}=(0.50,0.50,0.50,0.50,1)$ we have $I_{A}(\hat{\Pi}, c)=0$ and $I_{A}(\check{\Pi}, c)=1$. This result holds for any $c \in C$ since $I_{A}$ does not depend on $c$ explicitly. The index $I_{A}(.,$.$) is one such measure which preserves the$ $A F$ ordering. Below, we shall refer to (2.11) as the absolute value measure, and we shall write it more simply as $I_{A}(\Pi)$.

\section{A general class of measures}

There are several properties which we believe an inequality index may be required to satisfy in relation to SRHS data. We consider the following axioms:

- $C O N: I(\Pi, c)$ is a continuous function.

- $S C A L I N V: I\left(\Pi, c^{1}\right) \leq I\left(\Theta, c^{1}\right) \Longleftrightarrow I\left(\Pi, c^{2}\right) \leq I\left(\Theta, c^{2}\right)$ for any $c^{1}$ and $c^{2}$ $\in C$ and for any $\Pi, \Theta \in \Lambda$ (scale invariance).

- $\operatorname{NORM}: I(\Pi, c) \geq 0$ with $I(\hat{\Pi}, c)=0$ for any $c \in C$ (normalization).

- EQUAL : $\Pi \prec_{A F} \Theta \rightarrow I(\Pi, c) \leq I(\Theta, c)$ for any $c \in C$ (aversion to median preserving spreads).

The first axiom $C O N$ is a requirement that small changes in the distribution $\Pi$ and in the health scale $c$ do not produce large jumps in the value taken by the inequality index. The second axiom, $S C A L I N V$, requires that if two distributions exhibit the same level of inequality under a given health scale, the two distributions should be regarded as being equivalent under any alternative scale. Clearly, a weaker requirement would be that only under certain conditions the ordering of distributions be invariant to the choice of scale. We investigate in Section 5 below a weaker axiom of scale invariance defined along such lines.

The axiom NORM states that for any health scale, inequality is minimal if all the population is located at the median health state. Given this axiom, we normalize the minimum value taken by $I(\Pi, c)$ at zero. The fourth axiom is the core matter underlying the $A F$ ordering. EQUAL states that if $\Theta$ is a median preserving spread of $\Pi$, then it must be that $\Theta$ exhibits less equality than $\Pi$. 
There is an analogy to be drawn here with the Pigou-Dalton transfer principle underlying the income inequality literature (cf. Atkinson, 1970 and Sen, 1973) ${ }^{3}$. Assume that incomes are grouped into $n$ classes. Consider a Pigou-Dalton transfer from a person initially above the median to a person initially below the median income group. When such a transfer moves both individuals in the direction of the median, the transfer also achieves a reduction in inequality in the perspective of the $E Q U A L$ axiom underlying the $A F$ ordering.

Firstly, we examine the scale invariance axiom. It is useful at this stage to consider some general ordering relation $\prec_{\Lambda, C}$ defined in relation to an ordered pair $(\Pi, c)$. The scale invariance axiom implies that if for some scale $c^{1},\left(\Pi, c^{1}\right) \prec_{\Lambda, C}$ $\left(\Theta, c^{1}\right)$ then this result must hold true for any alternative scale $c^{2}$. If an ordering preserving function $f(\Pi, c)$ is to be constructed for the relation $\prec_{\Lambda, C}$, such that the property

$$
\left(\Pi, c^{1}\right) \prec_{\Lambda, C}\left(\Theta, c^{1}\right) \Longleftrightarrow\left(\Pi, c^{2}\right) \prec_{\Lambda, C}\left(\Theta, c^{2}\right)
$$

is satisfied, $f(\Pi, c)$ must be strictly separable in $\Pi$ (Blackorby et al., 1978; Theorem $3.2 \mathrm{a})$ :

Lemma 3 A function $f(\Pi, c)$ satisfies SCALINV if and only if it is of the form

$$
f(\Pi, c) \doteq \gamma[\Gamma(\Pi), c]
$$

where $\Gamma():. \Lambda \rightarrow R, \gamma():. \operatorname{ran}(\Gamma) \times C \rightarrow R$, and where $\gamma(.,$.$) is increasing in$ $\Gamma(\Pi)^{4}$.

Inequality indices such as the variance and coefficient of variation calculated using moments of the type $m\left(c^{\lambda}\right) \doteq \sum_{i=1}^{n} \pi_{i} c_{i}^{\lambda}$ where $\pi$ is a frequency distribution and $\lambda$ is a parameter, are not strictly separable in the sense of (3.2) ${ }^{5}$. Thus, in the present context where the health scale is an arbitrary ordered vector, Lemma 3 allows us to exclude such indices from the class of scale invariant measures.

In the result that follows, we characterize the family of inequality indices which satisfy the four axioms discussed above ${ }^{6}$.

\footnotetext{
${ }^{3}$ A Pigou-Dalton equalizing transfer between two individuals is such that (i) the sum of incomes before and after the transfer is unchanged and (ii) the distance between a poor and a richer person's income is reduced while the ranking of individuals is not reversed.

${ }^{4}$ Here, $\operatorname{ran}(f)$ denotes the range of a function $f$.

${ }^{5}$ Note that $\pi_{1}=\Pi_{1}$ and for $i>1, \pi_{i}=\Pi_{i}-\Pi_{i-1}$. The above $m\left(c^{\lambda}\right)$ moments are therefore functions of the cumulative distribution function.

${ }^{6}$ The proofs of Propositions 1 and 2, Lemma 4 and Corollary 2 are all presented in the Appendix.
} 
Proposition $1 I(\Pi, c)$ satisfies the axioms CON, SCALINV, NORM and $E Q U A L$ if and only if there exist continuous functions $\Delta: R_{+} \rightarrow R_{+}, \chi($.$) :$ $\operatorname{ran}(\Phi) \times C \rightarrow R$ and $\Phi():. \Lambda \rightarrow R$ such that

$$
I(\Pi, c) \doteq \Delta\{\chi[\Phi(\Pi), c]-\chi[\Phi(\hat{\Pi}), c]\}
$$

where $\Phi($.$) is an order preserving function for the relation \prec_{A F}, \chi($.$) is increasing$ in $\Phi(\Pi)$ and $\Delta$ is an increasing function such that $\Delta(0)=0$.

Though the result is proved in the Appendix, some intuition may be useful in interpreting this finding. Firstly, scale invariance places a separable structure on the function $\chi(.,$.$) requiring it to comply with the requirements of Lemma$ 3. Secondly, because for any $c$, inequality reaches its minimum value (zero) at $\hat{\Pi}, I(\Pi, c)$ must be a difference form as defined in (3.3). Thirdly, for $I(.,$.$) to$ satisfy the axiom $E Q U A L, \Phi(\Pi)$ must be an order preserving function for the $A F$ partial ordering. Note finally that if $I^{1}(\Pi, c) \doteq \chi[\Phi(\Pi), c]-\chi[\Phi(\hat{\Pi}), c]$, then for an increasing function $\Delta($.$) such that \Delta(0)=0, I^{2}(\Pi, c) \doteq \Delta\left[I^{1}(\Pi, c)\right]$ also satisfies the stated axioms in the Proposition. The index $I^{2}(\Pi, c)$ is however ordinally equivalent, in the sense of Cowell (2000, Section 4) to $I^{1}(\Pi, c)$.

To illustrate the result, consider the index

$$
\bar{I}(\Pi, c) \doteq\left(c_{1} c_{2} \cdots c_{n}\right)\left[1-\left(\frac{2 \sum_{i=1}^{n}\left|P_{i}-0.5\right|-1}{n-1}\right)\right]
$$

We can observe from our earlier Example 2 that $\bar{I}(\Pi, c) \doteq\left(c_{1} c_{2} \cdots c_{n}\right) \cdot I_{A}(\Pi)$. Accordingly, $\bar{I}(\Pi, c)$ may be shown to satisfy the normalization and aversion to median preserving spreads axioms ${ }^{7}$. It is also continuous in all of its arguments. To see that $\bar{I}(\Pi, c)$ also satisfies $S C A L I N V$, observe that for an alternative scale $b \in C$, we can write

$$
\bar{I}(\Pi, b) \doteq\left(\frac{b_{1} b_{2} \cdots b_{n}}{\left(c_{1} c_{2} \cdots c_{n}\right)}\right) \cdot \bar{I}(\Pi, c)
$$

Therefore, the new scale $b$ will preserve the ordering of two distributions $\Theta^{1}$ and $\Theta^{2}$ obtained under the original scale $c$.

\footnotetext{
${ }^{7}$ In particular, see Corollary 3 below.
} 
The index $\bar{I}(\Pi, c)$ may be viewed from the perspective of a wider family of indices written in the form ${ }^{8}$

$$
I(\Pi, c) \doteq \gamma\left[\left(\frac{\Phi(\Pi)-\Phi(\hat{\Pi})}{\Phi(\check{\Pi})-\Phi(\hat{\Pi})}\right), c\right]
$$

where $\Phi($.$) is as defined in the Proposition and \gamma($.$) is increasing in its first ar-$ gument. Because the function $\gamma($.$) of Lemma 3$ is strictly separable in $\Gamma(\Pi)$, it follows from Corollary 3.2.0a of Blackorby et al. (1978) that we can always construct some scale $c^{*} \in C$ such that $\gamma\left[\Gamma(\Pi), c^{*}\right]=\Gamma(\Pi)$. In the context of (3.4) for instance,

$$
c^{*} \doteq\left[c_{1} /\left(\prod_{i} c_{i}\right)^{1 / n}, \cdots, c_{n} /\left(\prod_{i} c_{i}\right)^{1 / n}\right]
$$

is such that $\bar{I}\left[I_{A}(\Pi), c^{*}\right]=I_{A}(\Pi)$.

The bottom line result from Proposition 1 and the above example is that the scale $c$ is an arbitrary reference vector for mapping $\Phi(\Pi)$ into an index $I[\Phi(\Pi), c]$. If we set $c \doteq c^{*}$ in relation to the family (3.3) we can construct a family of scale independent measures. This special case will provide the building block for the parametric family of indices we propose in the next section.

\section{A parametric family of indices}

Consider then adopting a scale $c^{*}$ in relation to the family (3.6), such that $I\left(\Pi, c^{*}\right)=[\Phi(\Pi)-\Phi(\hat{\Pi})] /[\Phi(\check{\Pi})-\Phi(\hat{\Pi})]$. Because then the inequality index does not depend explicitly on $c$, the resulting ratio form satisfies $C O N, E Q U A L$ and two stronger axioms:

- SCALINDEP : $I\left[\Pi, c^{1}\right]=I\left[\Pi, c^{2}\right]$ for any $c^{1}$ and $c^{2}$ elements of $C$ (scale independence).

- NORM2: $I(\Pi, c) \geq 0$ with $I(\hat{\Pi}, c)=0$ and $I(\check{\Pi}, c)=1$ (normalization).

These axioms are stronger in the sense that $S C A L I N D E P$ implies SCALINV and likewise NORM2 implies NORM :

\footnotetext{
${ }^{8}$ See in particular the developments in the next section, leading to Corollary 2.
} 
Corollary 1 Let $I(\Pi, c)$ be the family (3.6) defined in relation to any continuous order preserving function $\Phi(\Pi)$ for the relation $\prec_{A F}$. Then for $c \doteq c^{*}$, the resulting inequality index $I\left(\Pi, c^{*}\right)$ is of the form

$$
I^{*}(\Pi)=\frac{\Phi(\Pi)-\Phi(\hat{\Pi})}{\Phi(\check{\Pi})-\Phi(\hat{\Pi})}
$$

and satisfies the axioms CON, SCALINDEP, NORM2 and EQUAL.

The new scale invariance axiom SCALINDEP states that the inequality index takes on the same value for all increasing health scales. Thus, we can assign a maximum value 1 for the level of inequality underlying the distribution $\check{I}$. Note that in the context of $I^{*}(\Pi)$, the numerical scale plays no role in the measurement of inequality other than to order the health states from lowest to highest. This is the only information required to construct the cumulative distribution function.

Let $\Pi \doteq\left(P_{1}, \ldots, P_{n}\right)$ and $\Theta \doteq\left(Q_{1}, \ldots, Q_{n}\right)$ be two distributions with a common median $m$. The following result provides a building block in the direction of constructing inequality indices which preserve the ordering $\prec_{A F}$.

Lemma 4 Let $g_{1}(),. g_{2}():. R \longrightarrow R$ be any two continuous and increasing functions. Then $\Pi \prec_{A F} \Theta \Rightarrow \sum_{i<m} g_{1}\left(P_{i}\right) \leq \sum_{i<m} g_{1}\left(Q_{i}\right)$ and $\sum_{i \geq m} g_{2}\left(P_{i}\right) \geq$ $\sum_{i \geq m} g_{2}\left(Q_{i}\right)$.

Because the more egalitarian distribution $\Pi$ is required to have less mass below the median class but more mass above, the two functions $g_{1}$ and $g_{2}$ must order the vectors $\Pi^{L}$ and $\Theta^{L}$, and $\Pi^{H}$ and $\Theta^{H}$ oppositely. For instance, if we take $g_{1}()=.g_{2}()=.g($.$) , a continuous and increasing function, the lemma states that$ when $\Pi \prec_{A F} \Theta$ we have $\sum_{i<m} g\left(P_{i}\right) \leq \sum_{i<m} g\left(Q_{i}\right)$ and $\sum_{i \geqslant m} g\left(P_{i}\right) \geqslant \sum_{i \geqslant m} g\left(Q_{i}\right)$.

Observe that when $\Pi \prec_{A F} \Theta$, $\Pi^{L}$ dominates $\Theta^{L}$ in the sense of first order stochastic dominance, and likewise $\Theta^{H}$ dominates $\Pi^{H}$. As such, the functions $g_{1}($.) and $g_{2}($.$) need only be increasing, and convexity is not required. The convexity$ property of the underlying inequality index is clearly a property which features prominently in the income inequality literature in relation to the Pigou-Dalton transfer principle. Below however we shall choose $g_{1}($.$) and g_{2}($.$) as increasing and$ convex power functions for the purpose of making the index easily operational, not because convexity is required to preserve the relation $\prec_{A F}$. 
In particular, if we let $g_{1}(),. g_{2}($.$) be any two increasing and continuous func-$ tions and construct the $A F$ order preserving function as

$$
\Phi(\Pi) \doteq \sum_{i<m} g_{1}\left(P_{i}\right)-\sum_{i \geq m} g_{2}\left(P_{i}\right)
$$

then it follows that the resulting inequality index (4.1) will satisfy the axioms $C O N, S C A L I N V, N O R M$, and INEQ. The following result obtains from the application of Lemma 4 to Corollary 1 :

Corollary 2 The inequality index

$$
\begin{aligned}
I_{\alpha, \beta}(\Pi) & \doteq\left(\frac{\sum_{i<m} P_{i}^{\alpha}-\sum_{i \geq m} P_{i}^{\beta}+(n+1-m)}{k_{\alpha, \beta}+(n+1-m)}\right), \quad \alpha, \beta \geq 1 \\
k_{\alpha, \beta} & \doteq(m-1)\left(\frac{1}{2}\right)^{\alpha}-\left[1+(n-m)\left(\frac{1}{2}\right)^{\beta}\right]
\end{aligned}
$$

satisfies the axioms CON, SCALINDEP, NORM2, and INEQ.

The measure obtains by replacing in (4.2) the function

$$
\Phi_{\alpha, \beta}(\Pi) \doteq \sum_{i<m} P_{i}^{\alpha}-\sum_{i \geq m} P_{i}^{\beta}
$$

The parameter $k_{\alpha, \beta}$ is obtained via the relation $k_{\alpha, \beta}=\Phi_{\alpha, \beta}(\check{\Pi})$ and insures that the normalization axiom NORM2 is satisfied. Finally, the term $(n+1-m)$ appearing in the numerator and denominator of $(4.3)$ is $-\Phi_{\alpha, \beta}(\hat{\Pi})$.

There are two parameters $\alpha$ and $\beta$ introduced here in order to allow the researcher to accommodate differing judgments regarding inequality below and above the median. Clearly, $\Phi_{\alpha, \beta}($.$) satisfies the requirement of Lemma 4$ for all values $\alpha, \beta>0$. However, if say $0<\alpha<1$ while $\beta>1$ (or vice-versa) $g_{1}$ (.) becomes a concave function, while $g_{2}($.$) is convex. Then, establishing to which$ region of the distribution the index is sensitive becomes a tedious issue.

Consider then the context of the $\Phi_{\alpha, \beta}($.$) function where \alpha, \beta \geq 1$. For a given value of $\beta$, the inequality index becomes more sensitive to the cumulative probability mass at the bottom of the distribution as $\alpha \rightarrow 1$. Conversely, as $\alpha \rightarrow \infty$, 
the inequality index abstracts from the dispersion below the median:

$$
I_{\infty, \beta}(\Pi) \doteq\left(\frac{-\sum_{i \geq m} P_{i}^{\beta}+(n+1-m)}{-\left(1+(n-m)\left(\frac{1}{2}\right)^{\beta}\right)+(n+1-m)}\right), \quad \beta \geq 1
$$

As such, the views of a policy maker interested only in disparities at and above the median are accommodated by working with the index $I_{\infty, \beta}($.$) .$

To consider the second limiting scenario, where only inequality below the median is of interest, we derive the index $I_{\alpha, \infty}($.$) . We shall assume in what follows that$ $t$ is the number of classes $i$ for which $P_{i}=1$. We therefore have $1 \leq t \leq n-m+1$. Then, for a given $\alpha$, by choosing larger values for $\beta$ the inequality index becomes more sensitive to the mass at the top of the distribution, placing less weight on the values $P_{m}, \ldots, P_{n-t}$. At the limit, when $\beta=\infty$ we obtain for $1 \leq t \leq n-m+1$ the index

$$
I_{\alpha, \infty}(\Pi) \doteq\left(\frac{\sum_{i<m} P_{i}^{\alpha}-t+(n+1-m)}{-1+(m-1)\left(\frac{1}{2}\right)^{\alpha}+(n+1-m)}\right), \quad \alpha \geq 1
$$

which uses the dispersion below the median in the calculation of inequality. The final limiting case of interest we turn to below is the index $I_{1,1}(.)^{9}$.

The absolute value index (2.11) has been constructed using the form (4.3). Observing that $\left|P_{i}-0.5\right|=-\left(P_{i}-0.5\right)$ when $P_{i}<0.5$ and likewise, $\left|P_{i}-0.5\right|=$ $P_{i}-0.5$ when $P_{i} \geq 0.5$, we have the following result:

Corollary $3 I_{A}(\Pi)=I_{1,1}(\Pi)$.

The absolute value index is therefore the limiting case of the $I_{\alpha, \beta}($.$) family,$ when $\alpha=\beta=1$. The index is symmetric in the sense that equal deviations from 0.5 below and above the median are judged as being equivalent in terms of inequality.

${ }^{9}$ When both parameters approach infinity, inequality approaches the following constant:

$$
I_{\infty, \infty}(\Pi) \doteq 1-\frac{t-1}{(n-m)}, \quad 1 \leq t \leq n-m+1 .
$$




\section{A weaker axiom of scale invariance}

We have noted in the previous section that when $\Pi \prec_{A F} \Theta, \Pi^{L}$ dominates $\Theta^{L}$ and also $\Theta^{H}$ dominates $\Pi^{H}$ in the sense of first order stochastic dominance. Because the values $c_{i}$ attached to the various health states are ordered, we can use this additional information to propose alternative inequality indices for SRHS data. This is precisely the logic behind Theorem 3 of Allison and Foster (2004) as well as the measure $\omega(\pi ; c)$ these authors propose. For a frequency distribution $\pi \doteq\left(p_{1}, \ldots, p_{n}\right)$ and some numerical scale $c \in C, \omega(\pi ; c)$ is the difference between the average outcome above the median and the average outcome below the median:

$$
\omega(\pi ; c) \doteq\left(\frac{p_{m} c_{m}+p_{m+1} c_{m+1} \cdots+p_{n} c_{n}}{p_{m}+p_{m+1}+\cdots+p_{n}}\right)-\left(\frac{p_{1} c_{1}+p_{2} c_{2}+\cdots+p_{m-1} c_{m-1}}{p_{1}+p_{2}+\cdots+p_{m-1}}\right)
$$

As such, for a particular distribution $\Pi$, the index (5.1) will take on different values if applied to alternative scales. However, there are instances when the numerical values assigned to the scale will have no impact on the ordering of two distributions using the index $\omega(. ; c)$. It is precisely because when $\Pi \prec_{A F} \Theta, \Pi^{L}$ dominates $\Theta^{L}$ and also $\Theta^{H}$ dominates $\Pi^{H}$ in the sense of first order stochastic dominance, that the condition $\omega(\pi ; c) \leq \omega(\theta ; c)$ obtains for any scale $c \in C$.

It may thus be of interest to weaken our axiom of scale invariance so as to render the ordering of two distributions $\Pi$ and $\Theta$ invariant to the numerical scale when the condition $\Pi \prec_{A F} \Theta$ is satisfied:

- $W S C A L I N V: \Pi \prec_{A F} \Theta \rightarrow I(\Pi ; c) \leq I(\Theta ; c)$ for any scale $c \in C$.

Inequality indices that satisfy the axiom $W S C A L I N V$ need not satisfy the stronger axiom $S C A L I N V$. With some modification of the parametric form of the $I_{\alpha, \beta}($.$) family, we obtain the following family of indices to be used in conjunction$ with the axiom $W S C A L I N V$.

Proposition 2 The inequality index

$$
\begin{aligned}
J_{\alpha, \beta}(\Pi ; c) & \doteq\left(\frac{\sum_{i<m} c_{i} P_{i}^{\alpha}-\sum_{i \geq m} c_{i} P_{i}^{\beta}+\sum_{i \geq m} c_{i}}{\kappa_{\alpha, \beta}(c)+\sum_{i \geq m} c_{i}}\right), \quad \alpha, \beta \geq 1 \\
\kappa_{\alpha, \beta}(c) & \doteq \sum_{i=1}^{m-1} c_{i}\left(\frac{1}{2}\right)^{\alpha}-\sum_{i=m}^{n-1} c_{i}\left(\frac{1}{2}\right)^{\beta}-c_{n}
\end{aligned}
$$


satisfies the axioms CON, WSCALINV, NORM2, and INEQ for any scale $c \in C$.

Observe that the $J_{\alpha, \beta}(\Pi ; c)$ family does not belong to the class of indices discussed in Section 3 as it is not separable between the $A F$ order preserving function $\Phi_{\alpha, \beta}($.$) of (4.5) and the vector c$. This is why $J_{\alpha, \beta}(\Pi ; c)$ only satisfies $W S C A L I N V$ but fails to satisfy the stronger scale invariance axiom SCALINV.

\section{An illustrative application}

To illustrate the proposed methodology we use data from the 2002 wave of the Swiss Health Survey [SHS], conducted by Switzerland's Federal Statistical Office ${ }^{10}$. We have a total of 19706 observations from the seven statistical areas of Switzerland, in which respondents were asked to rate their health status between five possible health states ranging from very bad to very good (see Table 1 ).

It may be noted that the median health state is the fourth response $(m=4)$ in all seven distributions. The cumulative response at the median state good ranges between 0.72 in the Leman area and 0.87 in Ticino. Despite this finding, the $A F$ ordering only obtains in 6 out of the 21 pair-wise comparisons of Table 2 . Thus, for Central and Eastern Switzerland the SRHS distributions are unambiguously more egalitarian than the Leman data (fourth and sixth lines of column 2 of the Table). Likewise, Eastern Switzerland has less inequality than the Middle-Land. Because the Zurich distribution exhibits more equality than the East distribution, it follows from the transitive property of the relation $\prec_{A F}$ that the Zurich data are also more equally distributed than the Leman and Middle-Land data.

If a complete ranking of the seven distributions is required, inequality indices tailored for the $A F$ ordering may come handy. Because orderings are only available for one third of the pair-wise comparisons, it is necessary to compute a range of indices exhibiting sensitivity to different areas of the health distribution. Thus, in Table 3 we compute $I_{A}($.$) (the absolute value measure), I_{1,4}(),. I_{4,1}(),. I_{1, \infty}($. and $I_{\infty, 1}($.$) . There are several points which these results serve to illustrate.$

- All five inequality indices preserve the pair-wise ordering of distributions in the six cases where such comparisons can be made.

For instance, the indices for Zurich all indicate less inequality than the corresponding functions for the Leman data. This is not the case however when

\footnotetext{
${ }^{10}$ See Office Fédéral de la Statistique (2005) for a description of the survey.
} 
we compare Zurich and the North-West: inequality stands at 0.324 in Zurich and 0.335 in the North-West according to the $I_{1,4}($.$) measure, but inequality is lower in$ the North-West $(0.278$ versus 0.320$)$ according to the $I_{4,1}($.$) index. This example$ highlights a further point.

- Because orderings only obtain in one third of the pair-wise comparisons, the computation of inequality using a single index can be misleading.

As an additional example observe that the level of inequality underlying the East and Central distributions is identical (0.195) when assessed by means of the absolute value measure $I_{A}($.$) . Inequality is however lower in the East data (0.321$ vs. 0.350$)$ using the $I_{4,1}($.$) measure, but somewhat higher (0.468$ vs. 0.460$)$ on the basis of $I_{1, \infty}($.

The index $I_{\alpha, \beta}($.$) can be expressed as a function of I_{\alpha, \infty}($.$) and I_{\infty, \beta}($.$) . If we$ define the weights

$$
\begin{aligned}
& w_{\alpha} \doteq \frac{(n+1-m)+k_{\alpha, \infty}}{(n+1-m)+k_{\alpha, \beta}} \\
& w_{\beta} \doteq \frac{(n+1-m)+k_{\infty, \beta}}{(n+1-m)+k_{\alpha, \beta}}
\end{aligned}
$$

then we may readily obtain the following identity:

$$
I_{\alpha, \beta}(.)=w_{\alpha} I_{\alpha, \infty}(.)+w_{\beta} I_{\infty, \beta}(.)-\left(\frac{(n+1-m)-t}{(n+1-m)+k_{\alpha, \beta}}\right)
$$

- The computation of the indices $I_{1, \infty}($.$) and I_{\infty, 1}($.$) helps to characterize$ differences between the underlying distributions.

An inspection of the last column of Table 3 reveals that inequality at, and above, the median is highest in Leman $\left(I_{\infty, 1}()=0.560.\right)$ and lowest in Ticino $\left(I_{\infty, 1}()=0.26.\right)$. The $I_{1, \infty}($.$) measure however indicates that below the median$ inequality is higher in Ticino (0.496) than in Leman (0.488). Finally, the comparison of $I_{1, \infty}($.$) and I_{\infty, 1}($.$) in all seven distributions reveals clearly that it is in the$ better health states (the median and above) that the health distributions differ most.

The use of a parameter-free index such as the absolute value measure $I_{A}($. clearly commands the advantage of simplicity; however it may be of limited use in characterizing overall distributional differences in SRHS data. A parametric family of indices such as the $I_{\alpha, \beta}($.$) family we have proposed in this paper equips$ the data analyst with a more adequate tool for this latter purpose. 


\section{Appendix}

In this appendix we prove Lemma 4, Propositions 1 and 2 and Corollary 2. We begin with proofs of some preliminary results.

We first define a useful partial ordering relation. Let $x$ denote a vector in $R^{n}$ and define $D$ as a subset of $R^{n}$ such that $D \doteq\left\{x: x_{n} \geq x_{n-1} \geq \ldots \geq x_{1}\right)$.

Definition A1: If $x$ and $y$ are two elements of $D$, we shall say that $x \prec_{w} y$ $\Leftrightarrow \sum_{j=0}^{k} x_{n-j} \leq \sum_{j=0}^{k} y_{n-j}$ for all $k=0, \ldots, n-1$.

We next partition a distribution $\Pi \in \Lambda$ into two vectors $\Pi^{L}$ and $\Pi^{H}$ as in (2.2) and observe the following partial ordering relations when $\Pi \prec_{A F} \Theta$.

Lemma A1 Let $\Pi$ and $\Theta$ be two elements of $\Lambda$. Then $\Pi \prec_{A F} \Theta \Rightarrow \Pi^{L} \prec_{w} \Theta^{L}$ and $\Theta^{H} \prec_{w} \Pi^{H}$.

Proof. Since $P_{n} \geq P_{n-1} \geq \ldots \geq P_{1}$, we have that

$$
\begin{aligned}
P_{m-1} \leq & Q_{m-1} \\
P_{m-1}+P_{m-2} \leq & Q_{m-1}+Q_{m-2} \\
& \vdots \\
P_{m-1}+\cdots+P_{1} \leq & Q_{m-1}+\cdots+Q_{1}
\end{aligned}
$$

in other terms, $\Pi^{L} \prec_{w} \Theta^{L}$. Similarly,

$$
\begin{aligned}
Q_{n} & = \\
Q_{n}+Q_{n-1} \leq & P_{n}+P_{n-1} \\
& \vdots \\
Q_{n}+\cdots+Q_{m} \leq & P_{n}+\cdots+P_{m}
\end{aligned}
$$

which establishes that $\Theta^{H} \prec_{w} \Pi^{H}$. This completes the proof of the lemma.

Proof of Lemma 4. Since $\Pi \prec_{A F} \Theta$ entails $P_{j} \leq Q_{j}$ for all $j<m$, it follows when $g_{1}($.$) is a continuous increasing function that g_{1}\left(P_{j}\right) \leq g_{1}\left(Q_{j}\right)$ for all $j<m$. Hence $\sum_{i<m} g_{1}\left(P_{i}\right) \leq \sum_{i<m} g_{1}\left(Q_{i}\right)$.

Likewise, because $\Pi \prec_{A F} \Theta$ entails $P_{j} \geq Q_{j}$ for all $j \geq m$, it follows when $g_{2}($.$) is a continuous increasing function that we have \sum_{i \geq m} g_{2}\left(Q_{i}\right) \leq \sum_{i \geq m} g_{2}\left(P_{i}\right)$.

Proof of Proposition 1 First we prove that if $I(\Pi, c)$ satisfies the stated axioms, it must be of the form (3.3). 
Consider first the scale invariance axiom. If $I(\Pi, c)$ satisfies $S C A L I N V$, $I(\Pi, c)$ must be of the form stated in Lemma 3. Thus, $I(\Pi, c) \doteq \gamma[\Gamma(\Pi), c]$, where $\gamma($.$) is increasing in \Gamma($.$) .$

Together $S C A L I N V$ and $E Q U A L$ entail that $I(\Pi, c) \doteq \gamma[\Phi(\Pi), c]$ where $\Phi($. is an order preserving function for the relation $\prec_{A F}$, and where $\gamma($.$) is increasing$ in $\Phi($.$) .$

On the basis of Lemma 2 , for all $\Pi \in \Lambda$ we have $\hat{\Pi} \prec_{A F} \Pi$. Thus, $\Phi(\hat{\Pi}) \leq$ $\Phi(\Pi)$, and because $\gamma($.$) is increasing in its first argument, \gamma[\Phi(\hat{\Pi}), c] \leq \gamma[\Phi(\Pi), c]$. Thus, together SCALINV, EQUAL and NORM imply that either $(i) I(\Pi, c) \doteq$ $\chi[\Phi(\Pi), c]-\chi[\Phi(\hat{\Pi}), c]$ where $\chi(.,$.$) is increasing in \Phi(\Pi)$ and where $\Phi($.$) is an$ order preserving function for the relation $\prec_{A F}$, or $(i i) I(\Pi, c) \doteq \Delta\{\chi[\Phi(\Pi), c]-$ $\chi[\Phi(\hat{\Pi}), c]\}$, where $\Delta(0)=0$ and $\Delta: R_{+} \longmapsto R_{+}$is an increasing function. Finally, SCALINV, EQUAL, NORM and $C O N$ imply together that $\chi(.,),. \Phi($. and $\Delta($.$) must satisfy the above properties, and additionally must be continuous$ functions.

Conversely, let $I(\Pi, c)$ be of the form (3.3) where $\chi($.$) and \Phi($.$) are as stated$ in the Proposition. Then it can be verified straightforwardly that $I(\Pi, c)$ satisfies the axioms CON, NORM, SCALINV, and EQUAL.

Proof of Corollary 2 Let $g_{1}\left(P_{i}\right) \doteq P_{i}^{\alpha}$ and $g_{2}\left(P_{i}\right) \doteq P_{i}^{\beta}$. Then for $\alpha, \beta \geq 1$, $g_{1}($.$) and g_{2}($.$) are increasing continuous functions. Then, from Lemma 4 \Phi(\Pi)=$ $\sum_{i<m} P_{i}^{\alpha}-\sum_{i \geq m} P_{i}^{\beta}$ is an order preserving function for the relation $\prec_{A F}$. Furthermore because $g_{1}(0)=0$ and $g_{2}(1)=1, \Phi(\hat{\Pi})=-[n+1-m]$, while $\Phi(\check{\Pi})=(m-1)\left(\frac{1}{2}\right)^{\alpha}-$ $(n-m)\left(\frac{1}{2}\right)^{\beta}-1$. Therefore, defining

$$
\begin{aligned}
k_{\alpha, \beta} & \doteq(m-1)\left(\frac{1}{2}\right)^{\alpha}-(n-m)\left(\frac{1}{2}\right)^{\beta}-1 \\
I_{\alpha, \beta}(\Pi) & \doteq \frac{\sum_{i<m} P_{i}^{\alpha}-\sum_{i \geq m} P_{i}^{\beta}+(n+1-m)}{k_{\alpha, \beta}+(n+1-m)}
\end{aligned}
$$

It follows from Corollary 1 that $I_{\alpha, \beta}($.$) satisfies the four stated axioms.$

Proof of Proposition 2 Let $c \in C$. Then we have that $c_{n} \geq c_{n-1} \geq \cdots \geq$ $c_{1}>0$. From this it follows that for any $\beta \geq 1$

$$
\frac{\partial c_{n} P_{n}^{\beta}}{\partial P_{n}} \geq \frac{\partial c_{n-1} P_{n-1}^{\beta}}{\partial P_{n-1}} \geq \cdots \geq \frac{\partial c_{m} P_{m}^{\beta}}{\partial P_{m}}>0
$$


Likewise, for $\alpha \geq 1$

$$
\frac{\partial c_{m-1} P_{m-1}^{\alpha}}{\partial P_{m-1}} \geq \frac{\partial c_{m-2} P_{m-2}^{\alpha}}{\partial P_{m-2}} \geq \cdots \geq \frac{\partial c_{1} P_{1}^{\alpha}}{\partial P_{1}} \geq 0
$$

It follows from Marshall and Olkin (1979, Theorem 3.A.7) that $\Theta^{H} \prec_{w} \Pi^{H}$ implies that $\sum_{i \geq m} c_{i} P_{i}^{\beta} \geq \sum_{i \geq m} c_{i} Q_{i}^{\beta}$ for all $\beta \geq 1$. Likewise $\Pi^{L} \prec_{w} \Theta^{L}$ implies that $\sum_{i<m} c_{i} P_{i}^{\alpha} \leq$ $\sum_{i<m} c_{i} Q_{i}^{\alpha}$ for all $\alpha \geq 1$.

From Lemma A1, it follows therefore that $\Pi \prec_{A F} \Theta$ entails $\sum_{i \geq m} c_{i} P_{i}^{\beta} \geq \sum_{i \geq m} c_{i} Q_{i}^{\beta}$ for all $\beta \geq 1$, and likewise that $\sum_{i<m} c_{i} P_{i}^{\alpha} \leq \sum_{i<m} c_{i} Q_{i}^{\alpha}$ for all $\alpha \geq 1$. Therefore $J_{\alpha, \beta}(.,$.$) satisfies the axiom INEQ.$

Because $J_{\alpha, \beta}(.,$.$) satisfies the axiom I N E Q$ for any $c \in C$, it also follows that $\Pi \prec_{A F} \Theta \rightarrow J_{\alpha, \beta}\left(\Pi ; c^{1}\right) \leq J_{\alpha, \beta}\left(\Theta ; c^{1}\right)$ and $J_{\alpha, \beta}\left(\Pi ; c^{2}\right) \leq J_{\alpha, \beta}\left(\Theta ; c^{2}\right)$ for any numerical scales $c^{1}, c^{2} \in C$. Therefore, $J_{\alpha, \beta}(.$, .) satisfies the axiom WSCALINV.

The proofs of $C O N$ and NORM2 are shown as in Corollary 2.

\section{References}

Allison R. A. and Foster J. (2004): "Measuring Health Inequalities Using Qualitative Data", Journal of Health Economics 23, 505-524.

Apouey B. (2006): "La Mesure de la Polarisation de Santé Subjective", mimeo, Paris Sciences Economiques.

Atkinson A. (1970): "On the Measurement of Inequality", Journal of Economic Theory 2, 244-263.

Blackorby C., D. Primont and R. Russell (1978), Duality, Separability and Functional Structure: Theory and Economic Applications, New York, NorthHolland.

Cowell F. (2000): "Measurement of Inequality" in Atkinson A. and F. Bourguignon eds.: Handbook of Income Distribution, Amsterdam, Elsevier.

Marshall A. and I. Olkin (1979): Inequalities: Theory of Majorization and its Applications, San Diego, Academic Press.

Office Fédéral de la Statistique (2005): Enquete Suisse sur la Santé 2002: Plan d'Echantillonnage, Pondération et Estimation de la Précision, Neuchatêl, Publications de l'Office Fédéral de la Statistique.

Sen A. (1973): On Economic Inequality, Oxford, Clarendon Press. 
Strauss J. and D. Thomas (1998) "Health, Nutrition and Economic Development", Journal of Economic Literature 36, 766-817.

Wagstaff A., Paci P., van Doorslaer E. (1991): "On the Measurement of Inequalities in Health", Social Science and Medicine 33, 545-57. 
Table 1

\begin{tabular}{|c|c|c|c|c|c|}
\hline \multirow[t]{2}{*}{ Area } & \multicolumn{5}{|c|}{ SRHS Distribution } \\
\hline & Very bad & Bad & So so & good & Very good \\
\hline Leman & 0.01 & 0.05 & 0.16 & 0.72 & 1.00 \\
\hline North - West & 0.01 & 0.05 & 0.18 & 0.81 & 1.00 \\
\hline Central & 0.00 & 0.02 & 0.13 & 0.76 & 1.00 \\
\hline Middle - Land & 0.01 & 0.04 & 0.17 & 0.77 & 1.00 \\
\hline East & 0.00 & 0.03 & 0.14 & 0.78 & 1.00 \\
\hline Ticino & 0.01 & 0.06 & 0.17 & 0.87 & 1.00 \\
\hline Zurich & 0.00 & 0.03 & 0.13 & 0.78 & 1.00 \\
\hline
\end{tabular}


Table 2

Allison-Foster Type Inequality Orderings

\begin{tabular}{|c|c|c|c|c|c|c|c|}
\hline Leman & & $<>$ & $>$ & $<>$ & $>$ & $<>$ & $>$ \\
\hline NW & $<>$ & & $<>$ & $<>$ & $<>$ & $<>$ & $\langle>$ \\
\hline ML & $<>$ & $<>$ & $\langle>$ & & $>$ & $\langle>$ & $>$ \\
\hline $\mathbf{E}$ & $<$ & $<>$ & $<>$ & $<$ & & $<>$ & $>$ \\
\hline
\end{tabular}

Notes: $\mathrm{X}<\mathrm{Y}$ signifies that $\mathrm{X}<_{A F} \mathrm{Y}$

$\mathrm{X}<>\mathrm{Y}$ signifies that no ordering is available

$\mathrm{X}>\mathrm{Y}$ signifies that $\mathrm{Y}<_{\mathrm{AF}} \mathrm{X}$ 
Table 3

\begin{tabular}{|c|c|c|c|c|c|}
\hline \multirow[t]{2}{*}{ Area } & \multicolumn{5}{|c|}{ Inequality measure } \\
\hline & $I_{A}(\mathrm{II})$ & $I_{1,4}(\mathrm{II})$ & $I_{4,1}(\mathrm{II})$ & $I_{1, \infty}(\mathrm{II})$ & $I_{\infty, 1}$ (II) \\
\hline Leman & 0.250 & 0.390 & 0.408 & 0.488 & 0.560 \\
\hline North - West & 0.215 & 0.332 & 0.278 & 0.496 & 0.380 \\
\hline Central & 0.195 & 0.335 & 0.350 & 0.460 & 0.480 \\
\hline Middle - Land & 0.225 & 0.356 & 0.336 & 0.488 & 0.460 \\
\hline East & 0.195 & 0.328 & 0.321 & 0.468 & 0.440 \\
\hline Ticino & 0.185 & 0.274 & 0.190 & 0.496 & 0.260 \\
\hline Zurich & 0.190 & 0.324 & 0.320 & 0.464 & 0.440 \\
\hline
\end{tabular}




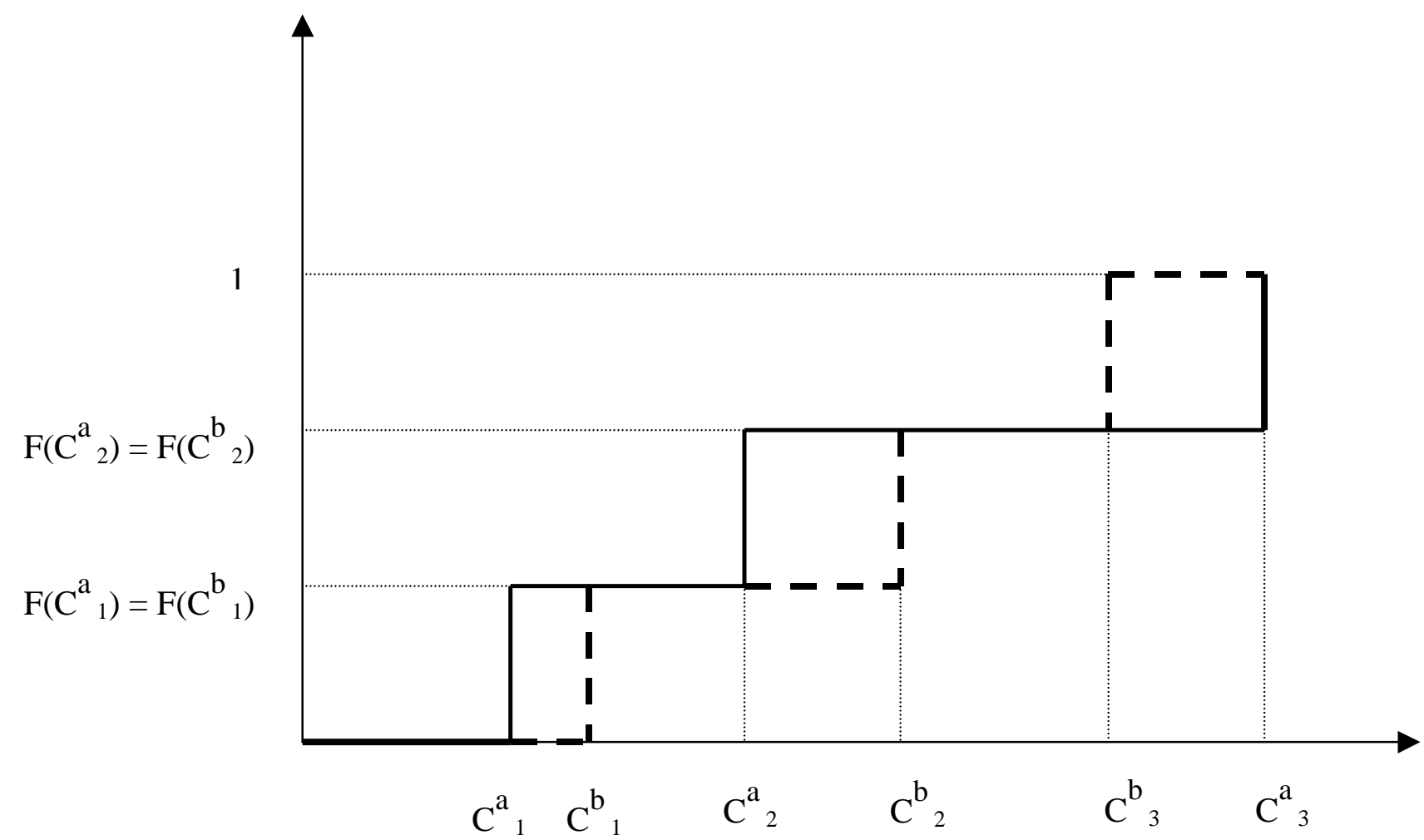

Figure 1: Changes in the health scale and the cumulative distribution function 